\title{
A Study of Evaluating Eye Movement Based on Ocular Motor Score for Children with Spastic Cerebral Palsy
}

\author{
Seo-Young Jeong', Tae-Young $\mathrm{Oh}^{2}$ \\ 'Department of Physical Therapy, Dongeui University Hospital, Busan, Republic of Korea; ${ }^{2}$ Department of Physical Therapy, College of Health and \\ Welfare, Silla University, Busan, Republic of Korea
}

Purpose: The purpose of this study is to introduce a tool for evaluating eye movement and analyze the reliability of measurement based on 10 cases of evaluating the oculomotor function of children with spastic cerebral palsy

Methods: The participants were selected by 6 medical and welfare institutions in Busan as GMFCS grade 1-3 among spastic diplegia and hemiplegia. Seven examiners evaluated 3 children for the evaluation of inter-rater agreement of Ocular Motor Score (OMS) and evaluated the condition of the ocular motor of 10 children using OMS, a re-examination was performed at six weeks after the initial examination. SPSS ver.25.0 was used to calculate the interclass correlation coefficient and Wilcoxon's signed-rank test, Mann Whitney-U test.

Results: The inter-rater agreement of OMS was 0.89 . Second mean values were decreased compared first examination in motility/ductions/version, saccades and smooth pursuit, but there was no significant difference. Children under 6 years old had a high mean value of saccades in first examination and the motility/ductions/version, fixation, saccades in second examination, but there was no significant difference. Spastic diplegia children's mean values were higher in head posture, fixation in 8 gaze directions than hemiplegia children in both first and second examination, but there were no significant differences.

Conclusion: Ocular motor function in 10 children of spastic children who participated in the study and could see that the scores was differed depending on age, type, grade of cerebral palsy. OMS may be available for this purpose.

Keywords: Eye movement, Spastic cerebral palsy, Interclass coefficient

\section{INTRODUCTION}

Cerebral palsy (CP) refers to a group of permanent movement disorders that affect a person's ability to move and maintain balance due to nonprogressive lesion of the developing brain of the fetus or infants. The movement disorders of cerebral palsy are accompanied by disturbances of sensation, perception, cognition, communication, behavior, epilepsy, and secondary musculoskeletal problems. ${ }^{1} \mathrm{CP}$ has been classified through traditional classification methods such as tension, movement disorder, the pattern of involvement, or the child's current function with regard to head and/or trunk control, independent sitting, and ambulation. ${ }^{2}$ The prevalence of $\mathrm{CP}$ was reported to be 2.11 per 1,000 live births, ${ }^{3}$ and the prevalence in Korea from 2004 to 2008 was 2.6 per 1,000 children (based on 5 -year-old patients). ${ }^{4}$

Children with cerebral palsy are often accompanied by a dysfunction of a visual system consisting of an anterior part-both eyes and the optic nerves up to the optic chiasm-and a posterior part-the optic tracts, the lateral geniculate nuclei, the optic radiations, and the occipital cortex. ${ }^{5}$ Among children with cerebral palsy, 49.6\% had ophthalmological disorders, ${ }^{6}$ and ocular motility and refractive errors were reported as the most common visual problems among children. In addition to problems related to refractive errors and ocular motility, some children had poor visual and perceptual performance, such as poor visual acuity, intermittent fixation, and lack of visual attention. This is regarded as cerebral visual impairment $(\mathrm{CVI})^{?}$

$\mathrm{CVI}$ is commonly defined as a disturbance of vision caused by a defective function of the retrochiasmatic part of the visual system in the absence of any critical ocular disease. ${ }^{8}$ As above, children with cerebral palsy experience a wide variety of visual problems including strabismus, refractive disorder, and retinopathy. Among these problems, eye movement dis-
Received Nov 11, 2020 Revised Dec 17, 2020

Accepted Dec 28, 2020

Corresponding author Tae-Young Oh

E-mail ohtaeyoung@silla.ac.kr
Copylight ( 2020 The Korean Society of Physical Therapy

This is an Open Access article distribute under the terms of the Creative Commons Attribution Non-commercial License (https:// creativecommons.org/license/by-nc/4.o.) which permits unrestricted non-commercial use, distribution, and reproduction in any medium, provided the original work is properly cited. 
orders are most common showing functional abnormalities such as gaze, saccade, and smooth pursuit in addition to strabismus and abnormal eye movements. In other words, the aspects of cerebral palsy are based not only on motor dysfunction characteristics and local anatomy but also on neuro-ophthalmology. ${ }^{9}$

Based on classifying children with spastic cerebral palsy according to the degree of motor impairment using the gross motor function classification system (GMFCS), Costa et al..$^{10}$ found a high correlation between the degree of motor impairment and visual loss using the sweep visual evoked potential method. The visual perception system has a great influence on postural control and motor development by engaging in spatial association, identifying object characteristics, and distinguishing objects and their backgrounds, ${ }^{7}$ and eye movement function to place the image of nearby objects in the center also acts as an important factor in postural control and motor development. ${ }^{11}$ Tools for evaluating the visual perception and visual-motor function of children include the development test of visual perception (DTVP), motor-free visual perception test (MVPT), and the test of visual perceptual skills (TVPS). Children with cerebral palsy attained significantly lower TVPS scores compared to normal children, ${ }^{12}$ and showed significant defects in visual-motor skills due to visual perception disorders. ${ }^{13}$

Eye movement control is the most basic type of visual perception, and lack of eye movement control often occurs in patients suffering from central nervous system diseases. ${ }^{14}$ Therefore, it is crucial to evaluate the gross motor function of children with cerebral palsy as well as to perform an early evaluation of visual problems, accurately detect visual impairments, and evaluate eye movement function, which is the basis of visual perception. However, despite the wide variety of studies on visual perception and visual-motor functions of children with cerebral palsy, there is still a lack of research on oculomotor function evaluation.

The purpose of this study is to introduce a tool for evaluating eye movement and analyze the reliability of measurement based on 10 cases of evaluating the oculomotor function of children with spastic cerebral palsy, and to provide the significance of eye movement in clinical practice by identifying the types of children with cerebral palsy and the characteristics of eye movement by each case.

\section{METHOD}

\section{Subjects and Period}

The 10 subjects were selected from six institutions that treat children with cerebral palsy in Busan, Republic or Korea. The research was conducted after explaining the purpose of this study to the parents of the children and obtaining consent to participate in the study. The subjects were selected according to the following criteria. 1) Children diagnosed with spastic diplegia or hemiplegia, 2) Children between the age of four and ten, 3) GMFCS level 1-3: capable of head control and independent sitting, 4) Children who can understand the examiner's instructions, 5) Children with parents who agreed to allow their children to participate in the study. The study was conducted from April 1 to May 31, 2019.

\section{Measurement}

This study used the GMFCS to evaluate the physical functions of the participants and the Ocular Motor Score (OMS) to evaluate the ocular motor function.

\section{1) Gross motor functional classification system}

The GMFCS divides children with cerebral palsy into four age groups (under 2 years, 2-4 years, 4-6 years, 6-12 years) and classifies the degree of disability into five levels for each age group. This five-level classification system was designed to reflect the differences in gross motor function that are meaningful in the daily lives of children with cerebral palsy and their families, with an emphasis on sitting and walking. A classification is made by determining which level best corresponds to a child's present gross motor function. The GMFCS is a reliable and valid classification method with high interrater reliability $(G=0.93)$ and test-retest reliability $(G=$ 0.79), so it was suitable for screening children for evaluation. ${ }^{15}$ The participants in this study had cerebral palsy corresponding to levels 1-3.

\section{2) Ocular motor score}

The OMS is a reliable tool for evaluating the ocular motor functions of children, which shows an observed intra-rater reliability of 0.88 and interrater reliability of $0.80 .{ }^{16}$ The OMS used in this study was translated into Korean after obtaining a consent from the author Monica Olsson ${ }^{16}$, to evaluate the ocular motor functions of Korean children with cerebral palsy.

This study selected and used 10 of the 15 items including of head posture, lid position, pupil response, strabismus, motility/ductions/versions, fixation in primary position, fixation in 8 gaze direction, saccades, smooth pursuit, convergence.

\section{Measurement procedures of OMS}

We translated 10 items of OMS into Korean and were supervised by a bi- 
lingual who spoke English and Korean. The term was standardized by 7 examiner who participated to this study.

To determine the test items used in this study, a group of physical therapists with more than three years of clinical experience chose the ocular movement score measurement items and unified the measurement method and tools. In terms of interrater reliability, seven examiners randomly selected and tested three children, and a second test was conducted six weeks after the first test to evaluate the ocular movement status of 10 children.

\section{Statistical analysis}

To assess the interrater reliability of OMS, seven examiners randomly selected and evaluated three children, and SPSS ver. 25.0 was used to calculate the interclass correlation coefficient. The Wilcoxon's signed-rank test (non-parametric statistical test) was used to compare the initial state of the children and the state after six weeks, the Mann Whitney-U test was performed to compare the ocular movement status according to age and types of cerebral palsy, and The Kruskal-Wallis test was used to compare the ocular movement status according to the GMFCS levels.

\section{RESULTS}

\section{The subjects' characteristics}

A total of 10 children (7 boys, 3 girls) participated in this study and their mean age was 6.3 years. There were three children in GMFCS level 1, three in level 2, and four in level 3. Seven children had spastic diplegia and three children had spastic hemiplegia.

\section{OMS inter-rater reliability test}

To assess the OMS inter-rater reliability, seven examiners tested three children and the observed reliability was 0.89 . A two-way mixed model was used as the intraclass correlation coefficient at a 95\% confidence interval to assess consistency.

\section{Comparison of OMS according to variations}

1) Comparing the first and second test results

A second test was performed six weeks after the first test to examine the children's eye movement status, and the Wilcoxon's signed-rank test was used to compare the test results. The mean values of motility, ductions, versions, saccades, and smooth pursuit decreased in the second test compared to the first test, but there was no statistical significance (Table 1).
Table 1. The comparison of OMS score between 1st and 2nd examination (unit: score)

\begin{tabular}{lccc}
\hline Items & 1st exam & 2nd exam & $\mathrm{p}$ \\
\hline Head posture & $0.20 \pm 0.26$ & $0.20 \pm 0.35$ & 1.00 \\
Lid position & $0,00 \pm 0.00$ & $0.00 \pm 0.00$ & 1.00 \\
Pupil response & $0.05 \pm 0.16$ & $0.10 \pm 0.21$ & 0.32 \\
Strabismus & $0.35 \pm 0.34$ & $0.35 \pm 0.34$ & 1.00 \\
Motility/Ductions/Nersions & $0.60 \pm 0.32$ & $0.50 \pm 0.33$ & 0.16 \\
Fixation in primary positions & $0.39 \pm 0.44$ & $0.35 \pm 0.37$ & 0.66 \\
Fixation in 8 gaze direction & $0.52 \pm 0.43$ & $0.48 \pm 0.37$ & 0.66 \\
Saccades & $0.55 \pm 0.40$ & $0.38 \pm 0.35$ & 0.10 \\
Smooth pursuit & $0.70 \pm 0.26$ & $0.60 \pm 0.21$ & 0.16 \\
Convergence & $0.35 \pm 0.47$ & $0.30 \pm 0.35$ & 0.66 \\
\hline
\end{tabular}

$p<0.05$.

\section{2) Comparison of OMS according to age}

In terms of comparing the test results according to the children's age, there were differences in the mean scores for saccade (children under 6: 0.65 \pm 0.40 , children 6 years or older: $0.48 \pm 0.42)$ and convergence $(0.25 \pm 0.50$, $0.42 \pm 0.49)$ in the first test, but there was no statistical significance.

In the second test, there were differences in the mean scores for motility/duction/version (children under 6: $0.63 \pm 0.25$, children 6 years or older: $0.42 \pm 0.38)$, visual fixation in eight gaze directions $(0.65 \pm 0.40,0.37 \pm$ $0.33)$, and saccade $(0.65 \pm 0.40,0.20 \pm 0.15)$, but there was no statistical significance (Table 2)

\section{3) Comparison of OMS according to GMFCS levels}

In terms of comparing the test results according to the GMFCS levels, there were differences in the mean scores for fixation in primary position (level 1: $0.43 \pm 0.51$, level 2: $0.10 \pm 0.17$, level 3: $0.58 \pm 0.51$ ) and visual fixation in eights gaze directions $(0.33 \pm 0.58,0.30 \pm 0.00$, $0.83 \pm 0.35)$ in the first test, but there was no statistical significance.

In the second test, there were differences in the mean scores for head position $(0.00 \pm 0.00,0.17 \pm 0.29,0.38 \pm 0.48)$, fixation in primary position $(0.43 \pm 0.51,0.10 \pm 0.17,0.48 \pm 0.35)$, and convergence $(0.33 \pm 0.29,0.00 \pm$ $0.00,0.50 \pm 0.41$ ), but there was no statistical significance (Tables 3,4 ).

\section{4) Comparison of OMS according to the types of cerebral palsy}

In terms of comparing the test results according to the types of cerebral palsy, there were differences in the mean scores for head position (diplegia: $0.29 \pm 0.27$, hemiplegia: $0.00 \pm 0.00)$ and visual fixation in eight gaze directions $(0.60 \pm 0.37,0.33 \pm 0.58)$, but there was no statistical significance.

In the second test, there were differences in head position $(0.29 \pm 0.39$, $0.00 \pm 0.00)$ and fixation in eight gaze directions $(0.60 \pm 0.37,0.20 \pm 0.17)$, 
Table 2. The comparison of OMS score between younger older group by 6 years old

(unit: score)

\begin{tabular}{|c|c|c|c|c|c|c|}
\hline \multirow{2}{*}{ Items } & \multicolumn{3}{|c|}{ 1st exam } & \multicolumn{3}{|c|}{ 2nd exam } \\
\hline & Under 6 yr & Over 6 yr & $\mathrm{p}$ & Under 6 yr & Over 6 yr & $\mathrm{p}$ \\
\hline Head posture & $0.25 \pm 0.29$ & $0.17 \pm 0.26$ & 0.76 & $0.13 \pm 0.25$ & $0.25 \pm 0.42$ & 0.76 \\
\hline Lid position & $0.00 \pm 0.00$ & $0.00 \pm 0.00$ & 1.00 & $0.00 \pm 0.00$ & $0.00 \pm 0.00$ & 1.00 \\
\hline Pupil response & $0.00 \pm 0.00$ & $0.08 \pm 0.20$ & 0.76 & $0.13 \pm 0.25$ & $0.08 \pm 0.20$ & 1.00 \\
\hline Strabismus & $0.38 \pm 0.48$ & $0.33 \pm 0.26$ & 1.00 & $0.38 \pm 0.48$ & $0.33 \pm 0.26$ & 1.00 \\
\hline Motility/Ductions/Versions & $0.63 \pm 0.25$ & $0.58 \pm 0.38$ & 1.00 & $0.63 \pm 0.25$ & $0.42 \pm 0.38$ & 0.48 \\
\hline Fixation in primary positions & $0.40 \pm 0.42$ & $0.38 \pm 0.49$ & 0.76 & $0.40 \pm 0.42$ & $0.32 \pm 0.37$ & 0.76 \\
\hline Fixation in 8 gaze direction & $0.58 \pm 0.51$ & $0.48 \pm 0.42$ & 1.00 & $0.65 \pm 0.40$ & $0.37 \pm 0.33$ & 0.35 \\
\hline Saccades & $0.65 \pm 0.40$ & $0.48 \pm 0.42$ & 0.61 & $0.65 \pm 0.40$ & $0.20 \pm 0.15$ & 0.11 \\
\hline Smooth pursuit & $0.75 \pm 0.29$ & $0.67 \pm 0.26$ & 0.76 & $0.63 \pm 0.25$ & $0.58 \pm 0.20$ & 1.00 \\
\hline Convergence & $0.25 \pm 0.50$ & $0.42 \pm 0.49$ & 0.61 & $0.25 \pm 0.29$ & $0.33 \pm 0.41$ & 0.91 \\
\hline
\end{tabular}

$p<0.05$.

Table 3. The comparison of 1st OMS score among each GMFCS grade

(unit: score)

\begin{tabular}{|c|c|c|c|c|}
\hline Items & Grade $1(n=3)$ & Grade $2(n=3)$ & Grade $3(n=4)$ & $\mathrm{p}$ \\
\hline Head posture & $0.17 \pm 0.29$ & $0.17 \pm 0.29$ & $0.25 \pm 0.29$ & 0.88 \\
\hline Lid position & $0.00 \pm 0.00$ & $0.00 \pm 0.00$ & $0.00 \pm 0.00$ & 1.00 \\
\hline Pupil response & $0.00 \pm 0.00$ & $0.00 \pm 0.00$ & $0.13 \pm 0.25$ & 0.47 \\
\hline Strabismus & $0.33 \pm 0.58$ & $0.50 \pm 0.00$ & $0.25 \pm 0.29$ & 0.52 \\
\hline Motility/Ductions/Versions & $0.50 \pm 0.50$ & $0.50 \pm 0.00$ & $0.75 \pm 0.29$ & 0.47 \\
\hline Fixation in primary positions & $0.43 \pm 0.51$ & $0.10 \pm 0.17$ & $0.58 \pm 0.51$ & 0.39 \\
\hline Fixation in 8 gaze direction & $0.33 \pm 0.58$ & $0.30 \pm 0.00$ & $0.83 \pm 0.35$ & 0.18 \\
\hline Saccades & $0.43 \pm 0.51$ & $0.77 \pm 0.40$ & $0.48 \pm 0.35$ & 0.50 \\
\hline Smooth pursuit & $0.83 \pm 0.29$ & $0.67 \pm 0.29$ & $0.63 \pm 0.25$ & 0.55 \\
\hline Convergence & $0.33 \pm 0.58$ & $0.17 \pm 0.29$ & $0.50 \pm 0.58$ & 0.73 \\
\hline
\end{tabular}

$p<0.05$.

Table 4. The comparison of 2nd OMS score among each GMFCS grade

(unit: score)

\begin{tabular}{|c|c|c|c|c|}
\hline Items & Grade $1(n=3)$ & Grade $2(n=3)$ & Grade $3(n=4)$ & $\mathrm{p}$ \\
\hline Head posture & $0.00 \pm 0.00$ & $0.17 \pm 0.29$ & $0.38 \pm 0.48$ & 0.37 \\
\hline Lid position & $0.00 \pm 0.00$ & $0.00 \pm 0.00$ & $0.00 \pm 0.00$ & 1.00 \\
\hline Pupil response & $0.17 \pm 0.29$ & $0.00 \pm 0.00$ & $0.13 \pm 0.25$ & 0.60 \\
\hline Strabismus & $0.33 \pm 0.58$ & $0.50 \pm 0.00$ & $0.25 \pm 0.29$ & 0.52 \\
\hline Motility/Ductions/Nersions & $0.50 \pm 0.50$ & $0.33 \pm 0.29$ & $0.63 \pm 0.25$ & 0.52 \\
\hline Fixation in primary positions & $0.43 \pm 0.51$ & $0.10 \pm 0.17$ & $0.48 \pm 0.35$ & 0.24 \\
\hline Fixation in 8 gaze direction & $0.43 \pm 0.51$ & $0.30 \pm 0.00$ & $0.65 \pm 0.40$ & 0.47 \\
\hline Saccades & $0.43 \pm 0.51$ & $0.43 \pm 0.51$ & $0.30 \pm 0.00$ & 1.00 \\
\hline Smooth pursuit & $0.67 \pm 0.29$ & $0.50 \pm 0.00$ & $0.63 \pm 0.25$ & 0.60 \\
\hline Convergence & $0.33 \pm 0.29$ & $0.00 \pm 0.00$ & $0.50 \pm 0.41$ & 0.15 \\
\hline
\end{tabular}

$p<0.05$.

but there was no statistical significance (Table 5).

\section{DISCUSSION}

Tools for evaluating the visual perception and visual-motor function of children include the DTVP, MVPT, and the TVPS, and many studies used these tools to compare the test results of children with cerebral palsy. According to a study by Lim et al. ${ }^{17}$ on the effect of an eye movement program (consisting of saccadic eye movement, pursuit eye movement, vestibulo-ocular movement, and vergence eye movement) on the postural control and visual perceptual ability of children with spastic cerebral palsy, the results showed significant increases in Gross Motor Function Mea- 


\begin{tabular}{|c|c|c|c|c|c|c|}
\hline \multirow{2}{*}{ Items } & \multicolumn{3}{|c|}{ 1st exam } & \multicolumn{3}{|c|}{ 2nd exam } \\
\hline & Diplegia & Hemiplegia & $\mathrm{p}$ & Diplegia & Hemiplegia & $\mathrm{p}$ \\
\hline Head posture & $0.29 \pm 0.27$ & $0.00 \pm 0.00$ & 0.18 & $0.29 \pm 0.39$ & $0.00 \pm 0.00$ & 0.38 \\
\hline Lid position & $0.00 \pm 0.00$ & $0.00 \pm 0.00$ & 1.00 & $0.00 \pm 0.00$ & $0.00 \pm 0.00$ & 1.00 \\
\hline Pupil response & $0.07 \pm 0.19$ & $0.00 \pm 0.00$ & 0.83 & $0.07 \pm 0.19$ & $0.17 \pm 0.29$ & 0.67 \\
\hline Strabismus & $0.29 \pm 0.27$ & $0.50 \pm 0.50$ & 0.52 & $0.29 \pm 0.27$ & $0.50 \pm 0.50$ & 1.00 \\
\hline Motility/Ductions/Versions & $0.57 \pm 0.19$ & $0.67 \pm 0.58$ & 0.67 & $0.50 \pm 0.29$ & $0.50 \pm 0.50$ & 1.00 \\
\hline Fixation in primary positions & $0.37 \pm 0.45$ & $0.43 \pm 0.51$ & 0.83 & $0.41 \pm 0.42$ & $0.20 \pm 0.17$ & 0.67 \\
\hline Fixation in 8 gaze direction & $0.60 \pm 0.37$ & $0.33 \pm 0.58$ & 0.27 & $0.60 \pm 0.37$ & $0.20 \pm 0.17$ & 0.18 \\
\hline Saccades & $0.60 \pm 0.37$ & $0.43 \pm 0.51$ & 0.52 & $0.36 \pm 0.30$ & $0.43 \pm 0.51$ & 1.00 \\
\hline Smooth pursuit & $0.71 \pm 0.27$ & $0.67 \pm 0.29$ & 0.83 & $0.57 \pm 0.19$ & $0.67 \pm 0.29$ & 0.67 \\
\hline Convergence & $0.36 \pm 0.48$ & $0.33 \pm 0.58$ & 1.00 & $0.29 \pm 0.39$ & $0.33 \pm 0.29$ & 0.83 \\
\hline
\end{tabular}

$p<0.05$.

sures (GMFM), Pediatric Balance Scale (PBS), Pediatric Reach Test (PRT), and most of the items of the Korean-Development Test of Visual Perception (K-DTVP), showing that the eye movement training program was effective in the postural control and visual perception of children with spastic cerebral palsy.

Lee $^{18}$ used the K-DTVP to investigate the effect of an eye movement program on the balance and gross motor function of children with spastic hemiplegia with no abnormalities in visual perception. You ${ }^{19}$ showed significant correlation between activity of daily living and gross motor function children with cerebral palsy. And the results showed significant increases in PBS and GMFM through the eye movement program, showing that the program had a very high therapeutic effect on the balance and gross motor function of children with spastic hemiplegia. Shin ${ }^{20}$ reported that treatment programs including neck and trunk stabilization exercises have a positive effect on improving the motor function (gross motor function, upper limb function), trunk balance, and visual perception of children with cerebral palsy.

Therefore, the motor functions of children with cerebral palsy such as gross motor function, balance, and posture control show a close relationship with visual functions such as visual perception and eye movement, so early evaluations of visual problems and motor functions are of great importance.

Visual perception tests play a significant role in the rehabilitation of children with cerebral palsy, so it is necessary to evaluate eye movement functions, which is the basis of visual perception abilities.

The main role of the ocular motor system is to adjust the position of the fovea at the center of the retina, and there are six eye movements to maintain the fovea to a particular spot accurately. These include saccade, to quickly move the fovea to change the point of fixation; smooth-pursuit, which are tracking movements of the eyes designed to keep a moving stimulus on the fovea; vergence, to align the fovea of each eye with targets located at different distances from the observer; vestibulo-ocular reflex, a reflex acting to stabilize gaze during head movement; optokinetic movement, to track a stimulus during continuous head rotation or movement; and fixation, to maintain the eyes in position while gazing at a stimulus without moving the head. ${ }^{21}$

Patients with these ocular motor function problems are usually evaluated by the clinical work of neurologists, ophthalmologists, optometrists, and orthoptists. Eye movements take place in different parts of the brain, and abnormal eye movements can give clues to pathologies and may be a source of important information in the subsequent course of the disease affecting the central or peripheral nervous system.

Nowadays, there are several advanced methods to record eye movements, such as Video-oculography (VOG), which records movements of both eyes using a head-mounted mask equipped with small cameras. Most of these methods require the patient's cooperation, so they are not always suitable for children with attention disorders or neurological deficits, and clinical practice performed in pediatric or general ophthalmology may not have access to advanced and expensive technologies found only in laboratories, and may require longer examination time. ${ }^{22}$

According to a study on the effects of postural movement normalization and eye movement program on the oculomotor function of children with cerebral palsy, the computer-assisted test results showed improvements in visual fixation, saccadic eye movement, and pursuit eye movement. Therefore, postural movement normalization and eye movement programs may be helpful to restore the physical function of children with 
cerebral palsy. ${ }^{23}$

In a study on the neuro-ophthalmological disorders in cerebral palsy in terms of ophthalmological, oculomotor, and visual aspects, eye movement was evaluated by fixation, smooth pursuit, saccadic movements, visual axis alignment for detecting strabismus using the Hirschberg test and cover test, extrinsic ocular motility (normal or altered in presence of a gaze palsy or eye muscle deficit), intrinsic ocular motility (pupil response), and abnormal eye movement. ${ }^{9}$

A study by Black ${ }^{24,25}$ on the visual disorders associated with cerebral palsy evaluated eye movement by testing saccadic movement, smooth pursuit, and strabismus by cover test in addition to testing visual acuity, visual field, pupil response, color matching ability, and refraction. A study on the ocular manifestations in children with cerebral palsy used the Snellen chart, Kay picture test, and Preferential Looking Chart to assess visual acuity, extraocular motility and strabismus, nystagmus, and refraction. ${ }^{26}$

A study by Ozturk et al. ${ }^{27}$ on the ocular disorders of children with spastic cerebral palsy conducted a full ophthalmologic examination including fundoscopy, orthoptic assessment (with prism cover test or Hirschberg and Krimsky tests), cycloplegic refraction, assessment of visual acuity, and binocular single vision (BSV).

A study by Chae et $\mathrm{al}^{28}$ on the effect of eye movement training program for postural control and visual perceptual of children with cerebral palsy, eye movement training program was effective in the improvement of postural control and visual perceptual.

As above, most of the tests on the ocular motor function of children with cerebral palsy measure each item separately because there are no systematic tools to evaluate the ocular motor function. However, this study was limited to children with spastic cerebral palsy, and there is a limit to generalize the results of this study because there were not enough participants for the OMS test.

\section{REFERENCES}

1. Rosenbaum P, Paneth N, Leviton A et al. A report: the definition and classification of cerebral palsy April 2006. Dev Med Child Neurol Suppl 2007;109:8-14.

2. Wood E, Rosenbaum P. The gross motor function classification system for cerebral palsy: a study of reliability and stability over time. Dev Med Child Neurol. 2000;42(5):292-6.

3. Oskoui M, Coutinho F, Dykeman J et al. An update on the prevalence of cerebral palsy: a systematic review and meta-analysis. Dev Med Child Neurol. 2013;55(6):509-19.

4. Park MS, Kim SJ, Chung CY et al. Prevalence and lifetime healthcare cost of cerebral palsy in South Korea. Health Policy. 2011;100(2-3):234-8.

5. Porro G, Van der Linden D, Van Nieuwenhuizen O et al. Role of visual dysfunction in postural control in children with cerebral palsy. Neural plasticity. 2005;12(2-3):205-10.

6. Minciu I. Clinical correlations in cerebral palsy. Maedica. 2012:7(4):31924.

7. Alimovic S. Visual impairments in children with cerebral palsy. Hrvatska rejija za rehabilitacijska istrazivanja. 2012;48(1):96-103.

8. Tomsak RL. Cerebral visual disturbance in infantile encephalopathy. Neurology. 1988;38(3):512.

9. Fazzi E, Signorin SG, Piana RL et al. Neuro-opthalmological disorders in cerebral palsy: opthalmological, oculomotor, and visual aspects. Dev Med Child Neurol. 2012;54(8):730-6.

10. Da Costa MF, Salomao SR, Berezovsky A et al. Relationship between vision and motor impairment in children with spastic cerebral palsy: new evidence from electrophysiology. Behav Brain Res. 2004;149(2):145-50.

11. Thomas NM, Bampouras TM, Donovan T et al. Eye movements affect postural control in young and older females. Front Aging Neurosci. 2016(8):1-11.

12. Menken C, Cermak SA, Fisher A. Evaluating the visual-perceptual skills of children with cerebral palsy. Am J Occup Ther. 1987;41(10):646-51.

13. Jung HW. The effects of visual perception training program on the visual perception and eye-hand coordination skills of children with spastic cerebral palsy. Dankook university. Dissertation of Master's Degree. 2007.

14. Koo BO, Bae SS. Kim HS et al. The effect of eye movement on balance iimprovement by plegia side of adult hemiplegic patient. J Kor Soc Phys Ther. 2002;14(4):1-19.

15. Palisano R, Rosenbaum P, Walter S et al. Development and reliability of a system to classify gross motor function in children with cerebral palsy. Dev Med Child Neurol. 1997;39(4):214-23.

16. Olsson M, Fahnehjelm KT, Rydberg A et al. Ocular motor score(OMS): a clinical tool to evaluating ocular motor functions in children. Intra rater and inter-rater agreement. Acta Ophthalmologica. 2015;93(5):444-9.

17. Lim AJ, Han SH, Kim SR et al. The effect of eye movement program on postural control and visual perceptual ability of children with spastic cerebral palsy. J Korean Soc Occup Ther. 2011;19(2):85-96.

18. Lee JY. The effects of eye movement exercises on balance ability and gross motor function for the children with hemiplegic cerebral palsy. Dankook university. Dissertation of Master's Degree. 2011.

19. You HJ, Park SW, Lee HS. Correlation between the activities of daily living assessment and gross motor function measures in children with spastic cerebral palsy. J Kor Phys Ther. 2015;27(6):425-9.

20. Shin JW. The effect of neck and trunk stabilization exercise on motor skills and balance and visual perception of children with cerebral palsy. Daegu university. Dissertation of Doctorate Degree. 2016.

21. Alpern M. Eye movement. In: Handbook Sensory Physiology, Berlin, Springer, 1972:303-30.

22. Olsson M, Fahnehjelm KT, Rydberg A et al. Ocular motor score a novel clinical approach to evaluating ocular motor function in children. Acta Ophthalmological. 2013;91(6):564-70.

23. Han DW, Kong NH. The effects of the postural movement normalization and eye movement program on the oculomotor ability of children with cerebral palsy. Phys Ther Korea. 2007;14(3):32-40.

24. Black PD. Ocular defects in children with cerebral palsy. Br Med J. 
1980;281(6238):487-8.

25. Black PD. Visual disorders associated with cerebral palsy. Br J Ophthalmol. 1982;66(1):46-52.

26. Marasini S, Paudal N, Adhikari P et al. Ocular manifestation in children with cerebral palsy. Optom Vis Perform. 2011;42(3):178-82.
27. Ozturk AT, Berk AT, Yaman A. Ocular disorders in children with spastic subtype of cerebral palsy. Int J Ophthalmol. 2013;6(2):204-10.

28. Chae JH, Choi WH, Chung SM. Effect of eye movement training program on postural control and visual perceptual of children with cerebral palsy: case report. Neurotherapy. 2020;24(2):47-56. 